\title{
Anabases
}

ANABASES Traditions et réceptions de l'Antiquité

4 | 2006

Varia

\section{Da Omero a Miyazaki. La mitologia classica Negli « anime » (e nei « manga ») giapponesi : spunti per una futura ricerca}

\section{Eugenio Amato}

\author{
(2) OpenEdition \\ Journals \\ Edizione digitale \\ URL: http://journals.openedition.org/anabases/3020 \\ DOI: 10.4000/anabases.3020 \\ ISSN: 2256-9421 \\ Editore \\ E.R.A.S.M.E.
}

\section{Edizione cartacea}

Data di pubblicazione: 1 ottobre 2006

Paginazione: 275-280

ISSN: 1774-4296

\section{Notizia bibliografica digitale}

Eugenio Amato, « Da Omero a Miyazaki. La mitologia classica Negli « anime » (e nei « manga ») giapponesi : spunti per una futura ricerca ", Anabases [Online], 4 | 2006, Messo online il 01 janvier 2012, consultato il 20 octobre 2019. URL : http://journals.openedition.org/anabases/3020 ; DOI : 10.4000/anabases.3020

Questo documento è stato generato automaticamente il 20 ottobre 2019.

(c) Anabases 


\title{
Da Omero a Miyazaki. La mitologia classica Negli « anime » (e nei « manga ») giapponesi : spunti per una futura ricerca
}

\author{
Eugenio Amato
}

Correva l'anno 1984, quando in Italia veniva trasmessa in prima visione sulle reti Mediaset l'edizione italiana (in 46 episodi) delle avventure della piccola Pollon,

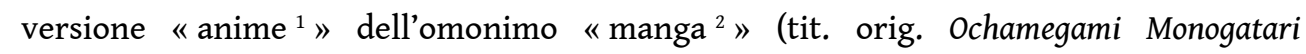
Korocoro Polon; trad. lett. Colocolo Polon, La storia della dea burlona), ideato da Azuma Hideo e prodotto nel 1979 dalla Kokusai Eiga ${ }^{3}$. Si trattava di un'animazione atipica, demenziale, ironica, irriverente e, soprattutto, educativa. Protagonista è, infatti, Pollon, piccola e vivace figlia di Apollo, dio del Sole, e nipotina del burbero e farfallone nonno Zeus. La bambina vive nell'Olimpo insieme ai suoi innumerevoli zii e cugini ed è immortale come tutte le divinità, ma malauguratamente, essendo troppo piccola, non è ancora una dea a tutti gli effetti, non avendo né titolo né poteri. È così che, un giorno, nonno Zeus, vedendo la nipotina affannarsi ostinatamente a diventare una vera dea, le dona uno speciale salvadanaio raffigurante un piccolo trono, da riempire con speciali monetine che egli stesso le attribuirà ad ogni buona azione commessa, sì da renderlo sempre più grande fino a quando la piccola non riuscirà a sedervi. In suo aiuto arriverà anche la Dea delle Dee, che guiderà ed aiuterà la giovane «apprendista » nelle sue avventure, facendole dono di un fermacapelli dotato di poteri speciali. Grazie ad esso, la giovane protagonista riuscirà a risolvere le più disparate situazioni in cui finirà per invischiarsi, tra i guai infiniti causati ai mortali, ma soprattutto agli abitanti stessi dell'Olimpo, personaggi a noi noti, talora, come immensamente malvagi, tremendi e maledetti, vissuti, tuttavia, come macchiette, caricature estreme di se stessi. Troviamo, ad esempio, Medusa ritratta come una deliziosa signorina che si è vantata troppo della sua bellezza, irritando così la «vecchia zitella antipaticona » Atena, la quale per punizione le restituirà una capigliatura rettile ed un aspetto mostruoso ; il «paparino » Apollo, il dio che conduce il carro del sole, con il piccolo problemino che alla mattina 
proprio non ne vuole sapere di alzarsi dal letto e, single incallito, preoccupato più delle donne che delle proprie responsabilità, se ne va cantando : « sono Apollo e mi trastullo, guido il carro e faccio il bullo "; le vendette di Era per gli innumerevoli tradimenti del «farfallone " nonno Zeus dalle connotazioni sadomaso (con tanto di frustino e calze a rete !) ; la stessa Pollon risolvere con estrema facilità l'enigma della Sfinge ed ancora rischiare per un fantasioso esperimento di far arrostire il padre Apollo con tutto il suo carro e sprofondare nelle tenebre l'intero cosmo. Nessuno dei personaggi che costellano l'universo mitico del monte Olimpo è assente : sfilano, dinanzi ai nostri occhi stupefatti e divertiti, Poseidone che non sa nuotare, Dafne in cerca di uno sposo, Atlante bisognoso d'aiuto, Artemide in sciopero, Cigno errante, Erisittone a dieta, Chimera in lacrime, Aracne alla tela, ecc. Nell'epilogo Pollon riesce addirittura a salvare la Terra dai mali usciti dal vaso di Pandora grazie alla speranza, ottenendo finalmente la realizzazione del suo ambito sogno : divenire lei stessa una dea, la Dea della speranza. Inseparabile compagno di avventure di Pollon (forse segretamente innamorato di lei) è il piccolo Eros, che da puttino dall'aspetto adorabile dell'iconografia classica è diventato una specie di mostriciattolo con le pulci nelle ali, un enorme ombelico sporgente al centro del ventre, e proprio lui non riesce a trovare l'amore.

2 Banalmente, il primo commento che verrebbe da fare è che C'era una volta... Pollon costituisca una parodia riduttiva dei miti greci. Ma a ben vedere l'olimpo è una società ricalcata su quella umana, con tanto di un corredo di vizi « da brivido » : dall'adulterio alla vendetta, dalla crudeltà alla gelosia, dalla pedofilia all'incesto. Al confronto, l'edulcorazione presente in Pollon non fa che nobilitare un mondo mitico che, almeno agli occhi di oggi, si rivela a dir poco agghiacciante. Ecco allora che, accanto ad un'interessante caratterizzazione dei personaggi, le più famose vicende greche vengono visitate in chiave leggera e divertente, senza rinunciare a una semplice, quanto efficace, intenzionalità educativa. Pollon certo non è un affidabile dizionario mitologico, ma ne è un potente alleato, una porta d'accesso ai miti greci o, se si vuole, una variante artistica. Basti anche solo immaginare a quanti discorsi sulla mitologia e sulle usanze greche possiamo fare a partire da Pollon. Se davvero, come genitori, come insegnanti, come educatori, siamo in grado di attuare operazioni di questo tipo, allora ben venga la mistificazione. Del resto, quale migliore bacino di collaudate risorse narrative da mettere in mano al cantore di oggi, ovvero la TV? In fondo si tratta pur sempre di raccontare allo scopo di divertirsi e, magari, di insegnare qualcosa ${ }^{4}$.

3 Non è questo, naturalmente, l'unico esempio di manga e di anime influenzati dalla cultura ed in particolare dalla mitologia classica. Anche in altri fumetti e serie animate giapponesi la cultura greca si ritaglia uno spazio ora dominante (basti citare, tra i vari titoli disponibili, produzioni quali Ulisse 31. La leggenda dello spazio [Uchu Densetsu Ulysses $31]^{5}$ ed Alexander. Cronache di guerra di Alessandro il Grande [Arekusandaa Senki ${ }^{6}$ ) ora più limitato, se non proprio semplicemente evocativo (si potrebbe citare, tra i numerosi esempi, la versione animata di uno dei celebri capolavori del grande regista e disegnatore Mayao Miyazaki, Nausicaa della Valle del vento [Kaze no Tani no Nausicaü] ${ }^{7}$, oppure, ai limiti del commerciale, la serie TV in 114 episodi di Saint Seiya [Seitoshi Seiya] ${ }^{8}$.

4 In quest'ultimo caso, infatti, gli spunti classici si nascondono dietro qualche singolo personaggio (molto spesso il solo nome) ovvero un'ambientazione esterna. Per quella che è forse la sua opera piú vasta e impegnativa (sette volumi nell'edizione italiana) - il racconto di un mondo post-tecnologico dove un'umanità respinta al margine dalla 
natura ostile e massicciamente inquinata cerca di sopravvivere, fra violenze tribali e scontri coi mostri che essa stessa ha creato -, Miyazaki dichiara espressamente di essersi ispirato per la sua protagonista Nausicaa all'omonimo personaggio dell'odissea di Omero, conosciuto inizialmente attraverso la lettura di una delle opere di Bernard Evslin (Heroes, gods and monsters of the Greek myths, New York 1967) ed apparso subito ai suoi occhi come il personaggio ideale per rivestire i panni di una giovane principessa candida e non-violenta, in intima sintonia con la natura, che porta il mondo alla salvazione e gli uomini alla conquista della libertà e della pace. Parimenti, la presenza del classico in Saint Seiya (vero rompicapo fantasy, collocato in un futuro molto prossimo, in cui s'immagina che dodici cavalieri, detti dello Zodiaco, combattano contro le nere forze del male in un torneo galattico organizzato da una certa Lady Isabel di Thule per il possesso dell'armatura d'oro di Sagitter) si limita ai soli nomi di taluni personaggi coinvolgendo, all'occorrenza, qualche ambientazione esterna: troviamo, ad es., Lady Pandora, Orfeo, Caronte, Niobe, Radamantis, Minos, Flegias ; la stessa lotta per il possesso delle armi d'oro del cavaliere stellare ricalca la lite scoppiata tra Ulisse e Aiace per le armi di Achille.

5 Nel primo caso, invece, l'influenza della civiltà greca è ben più schiacciante, se non proprio totalizzante, ma sempre sapientemente reinventata e reinterpretata cogli occhi di oggi. In Ulisse 31, ad es., l'omonimo eroe omerico, re di Itaca del XXXI secolo, è colto nel momento in cui riceve sulla base spaziale di Troia la notizia del forzato rientro in patria. Secondo un'antica legge del suo popolo, egli deve ripiegare verso casa prima dello scadere di dieci anni di assenza ; pena la perdita del trono. A bordo della sua nave Odissea, Ulisse fa così rotta verso casa, ma si imbatte in un pianeta dominato dal Ciclope, essere semidivino i cui adepti sono soliti sacrificargli dei giovani. Costoro rapiscono Telemaco, per salvare il quale Ulisse sarà costretto ad uccidere il Ciclope. Per tutta risposta, i fedeli servitori del terribile mostro galattico chiedono a Poseidone, dio del mare spaziale, di maledire Ulisse. Il suo equipaggio sarà condannato a restare sospeso nell'Ade, finchè il moderno eroe omerico non si recherà da loro in prima persona per riscattarli. Il cammino sarà, comunque, irto di difficoltà, riservando anche sorprese inattese, come l'incontro del protagonista col suo omonimo antenato greco: sarà questo lo spunto per esaltare lo spirito avventuriero di entrambi i personaggi, rimasto intatto nei millenni. Nella serie TV/OVA « Alexander » vengono, invece, trattate in chiave fantasy le vicende di Alessandro Magno, dal 338, anno della battaglia di Cheronea, al 326, data dello scontro contro re Poro sul fiume Jhelum. Sebbene l'anime, per l'originalità dell'ambientazione e del design, per l'estetica narrativa e le commistioni filosofico-matematiche che ne permeano la trama, si ponga nelle intenzioni degli autori come un'opera sperimentale ed alternativa, la ricostruzione degli avventimenti storici e, dunque, il rispetto della tradizione classica è più fedele ed attendibile di quanto ci si potrebbe aspettare, almeno nella sua cronologia essenziale. Naturalmente i caratteri dei personaggi sono strumentali e, in particolare i filosofi (Aristotele, Platone, Diogene e Pitagora), sono usati per il loro valore rappresentativo. Nel finale stesso, Aristotele ed Euclide fungono da simbolo di due mondi consequenziali, divisi da una concezione filosofico-matematica di diverso livello, il secondo destinato a sovrapporsi gradualmente al primo, da cui è derivato. Eccettuandone in alcuni la trasposizione metafisica (per esempio la battaglia contro Poro), i principali episodi storici sono esposti, pur nell'adattamento scenico, in modo tale da rimanere concilianti con la verità. Ne è un esempio la battaglia di Cheronea, dove realmente la cavalleria guidata da Alessandro giocò un ruolo fondamentale nel 
successo macedone; a quello scontro le fonti storiche attribuiscono il verificarsi, per la prima volta sul campo, della coordinazione tra falange e cavalleria, quella fusione organica e disciplinata tra forza d'urto e mobilità che rese successivamente formidabile l'esercito di Alessandro il Grande, e che fu alla base delle sue innumerevoli conquiste. La fedeltà storica può essere rintracciata anche in particolari che parrebbero a prima vista storicamente incongrui. Un esempio lo fornisce la ripetuta citazione delle Meraviglie del Mondo in numero di sette, apparentemente anacronistica dal momento che solo sei erano effettivamente esistenti al tempo di Alessandro (la costruzione del Faro di Alessandria fu, per ovvie ragioni, cronologicamente postuma ai fatti narrati). In realtà, il riferimento può rivelare un'accurata attenzione da parte degli autori. L'identità delle Meraviglie variò infatti nel corso dei secoli, ma il loro numero (sette) restò sempre immutato. Nel 332 a.C., anno in cui Alessandro raggiunse l'Egitto, il Palazzo di Babilonia e i Giardini Pensili costituivano Meraviglie separate. La successiva edificazione di Alessandria d'Egitto determinò il loro accorpamento, per preservare appunto il numero sette nonostante l'ingresso del celebre Faro. Fondamentalmente corretta anche la rappresentazione dell'assassinio di Filippo II, che, nella realtà storica, avvenne nel contesto dei festeggiamenti per le nozze di una delle sue figlie con Alessandro principe d'Epiro. Nell'occasione, sfilarono realmente le tredici statue (12+1), mentre Attalo fu effettivamente ucciso per ordine di Alessandro, ed Euridice per ordine di Olimpiade. Contengono elementi verosimili anche molte delle libere interpretazioni proposte nella finzione scenica, prima fra tutte la pratica di riti cabirici all'interno della corte macedone. Tra i fatti non corrispondenti, va invece ricordata la morte di Dario, che non avvenne a Gaugamela, ma in un contesto successivo, a seguito del tradimento di uno dei suoi consiglieri (Besso di Battriana). Inoltre il rientro dall'India fu determinato dal rifiuto dell'esercito macedone di proseguire oltre, mentre Alessandro morì a Babilonia, sulla via del ritorno. Alcune inesattezze si riscontrano anche nei rapporti descritti tra Aristotele e Platone, in particolare riguardo i tempi e i motivi per i quali lo Stagirita lasciò l'Accademia.

Questi ora citati sono solo alcuni tra i numerosi esempi di riuso ed originale riappropriazione del patrimonio storico-mitologico di Grecia antica nell'immaginario fumettistico e cinematografico giapponese. Si tratta in ogni caso di citazioni profondamente sconcertanti, per almeno due ragioni. Prima di tutto perchè ci vengono da fonti inaspettate, da fuori quella civiltà «occidentale » che secondo la piú comune retorica ha nei Greci le sue radici comuni. Ma anche perché, prelevando dal compatto tessuto, ad es., della narrazione omerica i nomi e l'ethos di Nausicaa, di Polifemo e di Odisseo per trapiantarli in ambiti cosí diversi e cosí lontani, « esse sembrano ricalcare puntualmente la norma implicita in tanto citazionismo post-moderno, la segmentazione dell'antico in unità minime decontestualizzate e riusabili, sconnessi frammenti da rimontare ad arbitrio per innestarli altrove». Tanto piú pronti al riuso, anzi, quanto piú esso sia gratuito, senza implicare affatto quello statuto paradigmatico dell'antichità «classica » che per secoli ne ha assicurato nella tradizione europea la conoscenza e lo studio.

$7 \mathrm{Ma}$ - occorre chiedersi con Salvatore Settis ${ }^{9}$ - è davvero cosí ? Omero è davvero piú « nostro » che dei Giapponesi o dei musulmani ? Non dovremmo sbalordirci, piuttosto, per quanto quelle citazioni che vengono da cosí lontano sanno essere intense, appropriate, efficaci ? O forse citazioni come queste (e sarebbe facile allungarne la lista) appartengono ormai a un assai piú vasto orizzonte "globale ", nel quale l'antichità greca deve avere un suo piccolo posto in mezzo a tante altre antichità, indiane, cinesi, 
maya, tutte egualmente legittime come altrettanti, equivalenti serbatoi di nomi, aneddoti, «storie ", citazioni, curiosità ?

8 Sono interrogativi importanti, ai quali si potrà rispondere solo dopo un attento ed adeguato studio della ricezione del mondo classico negli «anime» (e nei «manga») giapponesi, finora, invece, del tutto eluso. Mentre, infatti, la presenza dell'antico nei fumetti occidentali e l'uso didattico che se ne può fare sono stati variamente trattati ${ }^{10}$, per il versante « anime » tutto o quasi è ancora da fare.

\section{NOTE}

1. Il termine " anime", derivato dall'inglese " animation ", è un neologismo giapponese, nato tra il 1977 ed il 1983 ed impiegato per indicare i cartoni animati nipponici, tratti in genere dai manga di successo, non solo quelli realizzati per le serie televisive o per l'home-video, ma anche per il cinema. In precedenza, si utilizzavano perifrasi quali manga eiga ("film di manga ") e TV manga (" manga televisivo") : vedi F. PRANDONI, Anime al cinema. Storia del cinema d'animazione giapponese 1917-1995, Milano 1999, p. 74.

2. Il termine, coniato nel 1814 da Hokusai Katsushika, uno degli esponenti del cosiddetto genere Ukiyo-e ("Dipinti del mondo fluttuante") diffusosi a Tokyo dalla fine del XVII alla metà del XIX secolo (cf. Eureka! 11/12, Milano 1983, p. 4-5), designa i fumetti nipponici.

3. Per i dati tecnici (produttori, regia, animazioni, ecc.), vedi J. CLEMENTS - H. MCCARTHY, The Anime Encyclopedia. A Guide to Japanese Animation since 1917, Berkeley 2001, p. 303. In Francia la serie esce nel 1989 su La Cinq col titolo La petite Olympe et les dieux.

4. Cf. F. FILIPPI, in M. PELLITTERI, Mazinga Nostalgia. Storia, valori e linguaggi della Goldrake-generation, Roma $2002^{2}$, p. 374-375.

5. Per la scheda tecnica e la storia di tale anime, serie televisiva in 26 episodi apparsa in Giappone nel 1981, vedi CLEMENTS - MCCARTHY, op. cit., p. 420, i quali ne parlano come di "a ridiculously contrived sci-fi reworking of Homer's Odyssey". Si tratta della prima coproduzione francogiapponese, andata in onda in Francia tra il 1981 ed il 1986 inizialmente su RTL e, quindi, su France 3 (all'epoca France Régions 3), che ha visto tra i suoi ideatori Jean Chalopin (autore di Les mystérieuses cités d'or e dell'Inspecteur Gadget) e Nina Wolmark (autrice di Les Mondes Engloutis). Su tale anime esiste un aggiornato e quanto mai ricco sito elettronico, consultabile al seguente indirizzo : http ://ulysse31.saitis.net/.

6. Per la scheda tecnica e la storia di questa serie televisiva, tratta da un romanzo di Hiroshi Aromata e composta di 13 episodi, il primo dei quali trasmesso in Giappone nel dicembre del 1999, vedi nuovamente CLEMENTS - MCCARTHY, op. cit., p. 10. In Italia, essa è stata trasmessa dall'emittente televisiva MTV ed edita in 4 dvd dalla Dynamic.

7. Per la scheda tecnica e la storia di questa importante produzione cinematografica del 1984, tratta dal maga omonimo di Hayao Miyazaki e mandata in onda in Italia su RAI Uno nel 1987, vedi come sempre CLEMENTS - MCCARTHY, op. cit., p. 270.

8. Nota in Italia anche come I cavalieri dello Zodiaco, la serie, figlia dell'omonimo manga di Masami Kurumada, apparve per la prima volta in Giappone nel 1986 : vedi la scheda tecnica in CLEMENTS MCCARTHY, op. cit., p. 338-339. 
9. Vedi S. SETTIS, «Premessa», in ID. (a cura di), I Greci. Storia Cultura Arte e Società, 3. I Greci oltre la Grecia, Torino 2001, p. XXXIII-XXXVIII : XXXIII-XXXIV.

10. Cf. K. GEUS, M. HAASE, B. EICKHOFF, “Comics”, in Der Neue Pauly 3 (1999), col. 656-674 ed in particolare N. M. S. RODRIGUES, "A Antiguedade Clássica em Banda Desenhada”, in P. Cr. BARATA DIAS - J. RIBEIRO FERREIRA (edd.), Som e imagem no ensino das línguas clássicas. Actas, Coimbra 2003, p. 51-81 ; ID., "Apuleio, Fellini e Manara”, in Boletin de Estudos Clássicos 34 (2000), p. 161-172 ; ID., “ Evocações de Teseu e o Minotauro em BD", ibid. 41 (2004), p. 73-81; ID., "Da Odisseia à Eneida. Novos temas classicos em banda desenhada", ibid. 44 (2005), p. 153-180. Cf. inoltre A. VAN PRAET, “ Appropriation d'un mythe par le cinéma et la BD. La fin d'Atlantide", in BARATA DIAS - RIBEIRO FERREIRA, Som e imagem, cit., p. 83-92 e L. DA SILVA FERNANDES, "O Mundo greco-romano nas aventuras de Alix", ibid., p. 93-114.

\section{AUTORE}

\section{EUGENIO AMATO}

Département de Sciences de l'Antiquité

Université de Fribourg (Suisse)

eugenio.amato@unifr.ch 